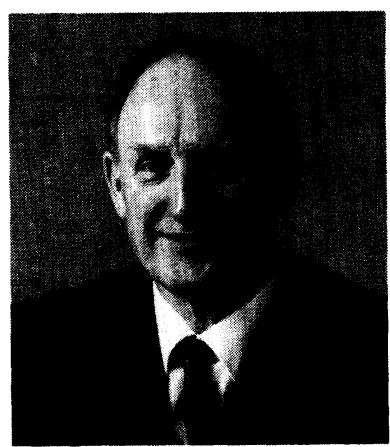

\section{The Year 1992 in Review: Part II}

In last month's editorial I gave some of the statistics regarding Volume 31 of Optical Engineering, published in 1992. When I did a similar review last year, I included a chart of the number of technical pages published per year since 1978. In this editorial, this chart is updated with the 1992 datum point (see Fig. 1).

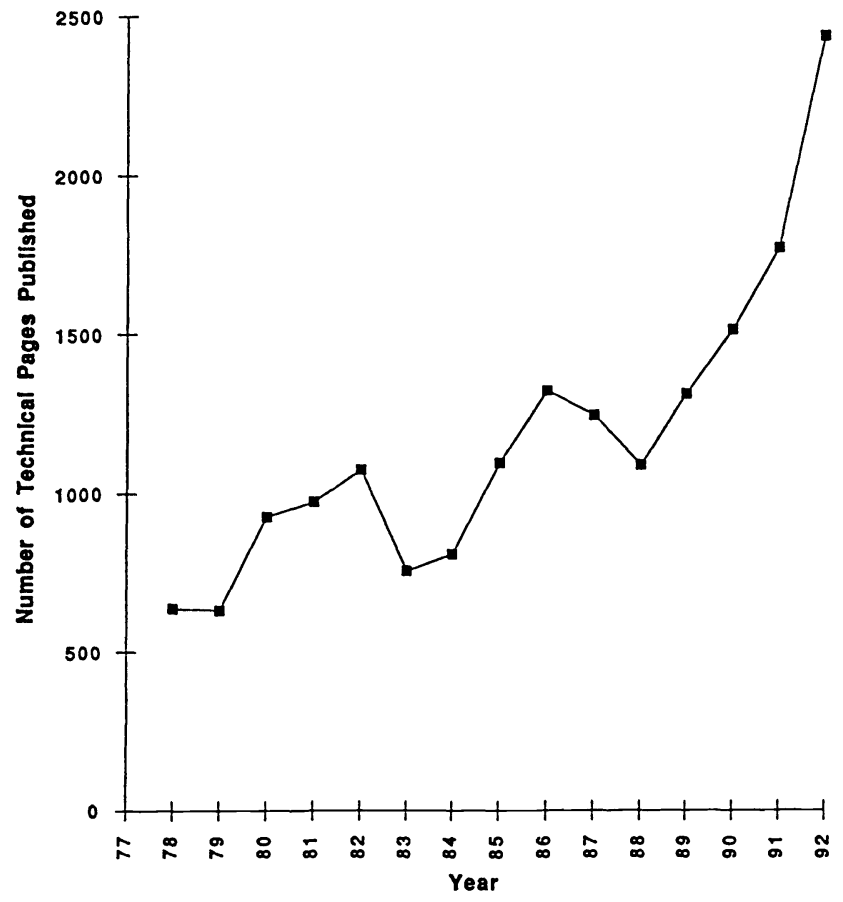

Fig. 1 The number of technical pages published from 1978 through 1992.

A significant number of the papers published in 1992 were papers received at our editorial office starting in December 1990. Through the end of 1991 I had received 242 papers, all of which have completed the review process. Readers might be interested in the following results.

$\begin{array}{lr}\text { Published } & 64.0 \% \\ \text { Rejected } & 26.4 \% \\ \text { Closed } & 7.0 \% \\ \text { Withdrawn } & 1.3 \% \\ \text { Transferred } & 1.3 \%\end{array}$

The "closed" papers are those that were returned to the authors for revision, and after a reasonable interval of time and several reminders, if I've heard nothing I finally close the files. I then treat any resubmission of the papers as new submissions.

\section{Papers Received in 1992}

We again had a record number of papers submitted to the journal during 1992 - a total of 311. In addition, 167 papers were received for special sections of the journal. Figure 2 shows a chart of the cumulative number of papers received by month for the last four years, exclusive of papers for special sections. The year 1992 shows a $35.8 \%$ increase over 1991.

Listed here are some of the important measures of activity that we track:

$\begin{array}{lrr}\text { Papers received } & 311 & +35.8 \% \\ \text { Number of reviewers } & & \\ \quad \text { solicited } & 801 & +60.5 \% \\ \text { Reviews received } & 468 & +44.0 \% \\ \text { Revised manuscripts } & & \\ \quad \text { received } & 160 & \\ \text { Papers accepted } & 192 & +137.0 \% \\ \text { Papers rejected } & 61 & +2.0 \% \\ \text { Papers withdrawn } & 7 & \\ \text { Papers closed } & 17 & \\ \text { Papers transferred } & 5 & \\ \text { Papers retured } & \end{array}$

Papers returned to authors for revision 


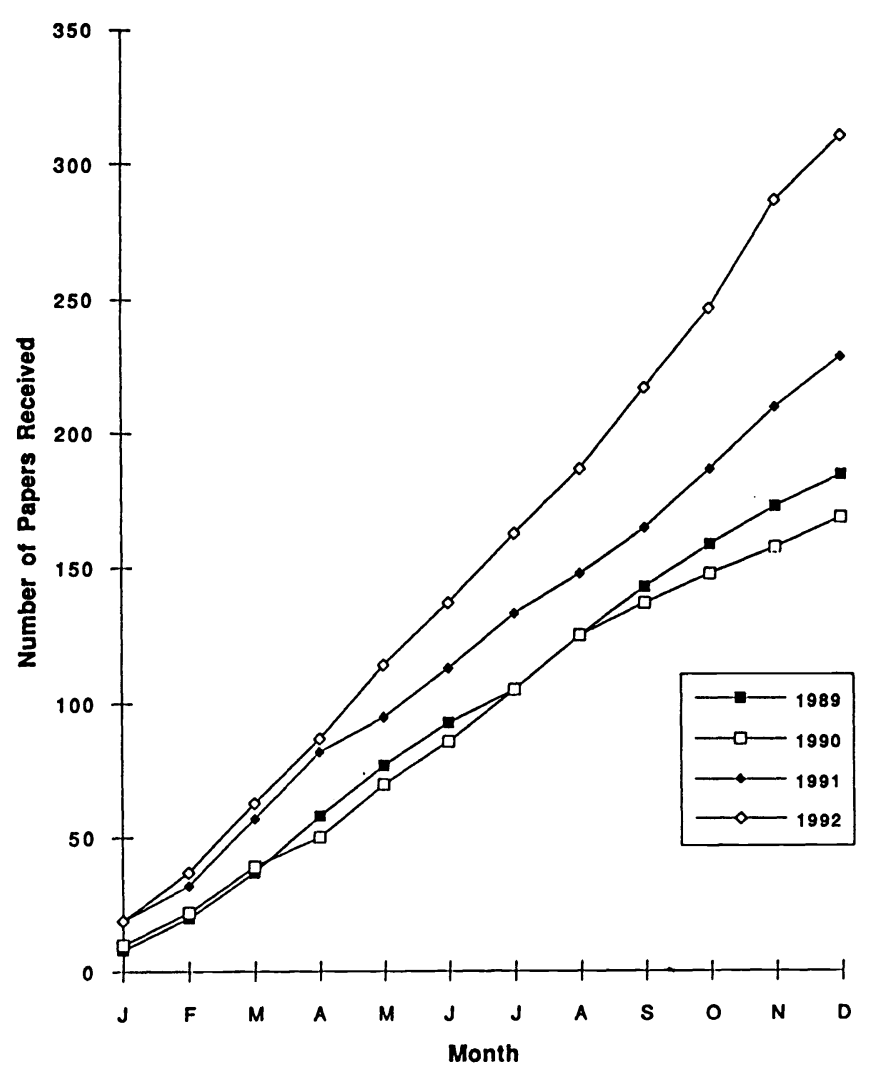

Fig. 2 The cumulative number of papers received each year for the years 1989 through 1992, exclusive of special-section papers.

\section{Status of Papers Received in 1992}

The following summarizes the current status of the 311 papers received during 1992:

$\begin{array}{lr}\text { Published } & 18 \\ \text { Accepted and in press } & 94 \\ \text { Rejected } & 44 \\ \text { In review } & 91 \\ \text { In revision } & 52 \\ \text { Withdrawn } & 6 \\ \text { Closed } & 3 \\ \text { Transferred } & 3\end{array}$

Volume 32-1993

As you know, this year's volume of Optical Engineering is already well under way. We certainly expect to see the activity level of your journal continue to increase during 1993, but not with the same percentage increase as last year (please!).

\section{Biomedical Optics Special Section}

Included in this issue is the second of two planned biomedical optics special sections of Optical Engineering-the first appeared in the July 1992 issue. We would like to thank Abraham Katzir for initiating these special sections and a special thanks goes to the following members of the review panel that coordinated the paper review process: Dr. Lawrence S. Bass, New York University Medical Center; Prof. James Harrington, Rutgers University; Dr. Douglas Johnson, University of Texas; Dr. Joseph Lakowicz, University of Maryland School of Medicine; Dr. Raymond J. Lanzafame, University of Rochester School of Medicine; Dr. Thomas Mang, Roswell Park Cancer Institute; Prof. Sohi Rastegar, Texas A\&MUniversity; Dr. Eva Sevick, Vanderbilt University; Dr. Bruce Tromberg, University of California at Irvine; and Dr. Gerald Vurek, Abbott Laboratories.

Brian J. Thompson Editor 
April 1993

Emerging Optoelectronic Technologies

Vijai K. Tripathi

Oregon State Univeristy

Dept. of Electrical and Computer Eng.

ECE Building 220

Corvallis, Oregon 97331-3211

$503 / 737-3617 \cdot 503 / 737-1300 \mathrm{FAX}$

\section{May 1993}

Phase Contrast Microscopy

Maksymilian Pluta

Institute of Applied Optics

ul. Kamionkowska 18

03-805 Warszawa, Poland

$4822184405 \cdot 4822133265$ FAX

June 1993

From Numerical to Symbolic Image

Processing: Systems \& Applications

G. Vernazza

Dipartimento di Ingegneria Biofisica ed

Elettronica

Universita degli Studi di Genova

Via Opera Pia, 11a

16145 Genova, Italy

+3910 353-2755 - +3910 353-2777 FAX

July 1993

Visual Communication and Image

Processing IV

Cheng-Tie Chen

Bellcore

445 South St.

Morristown, NJ 07962

201/829-5151 • 201/829-5884 FAX

Hsueh-Ming Hang

Center for Telecommunication Research

National Chiao-Tung University

Hsinchu, Taiwan

$+886 / 35-712121 \times 3298 \cdot+886 / 35-723283$ FAX

Kou-Hu Tzou

COMSAT Labs.

22300 Comsat Drive

Clarksburg, MD 20871

301/428-4663 - 301/428-7747 FAX

September 1993

Optical Science and Engineering in Canada

C.P. Grover

National Research Council

Institute for National Measurement Standards

Ottawa, Canada K1A OR6

613/993-2098 • 613/952-1394 FAX

Optical Engineering in Hungary

Tivadar Lippenyi

HUNGOPTIKA

Tartsay u. 24

Budapest H-1120, Hungary

$3611563985 \cdot 3611563985$ FAX

Zoltan Fuzessy

Technical Univ. Budapest

Department of Physics

Balazs Bela u.36.IV.8.

Budapest H-1094, Hungary

$3611666361 \cdot 3611666808$ FAX

October 1993

Microlithography

James R. Sheats

Hewlett-Packard Company

2500 Deer Creek Road
Palo Alto, CA 94304-1392

415/857-5987 • 415/857-6241 FAX

Manuscripts due March 1, 1993.

November 1993

Acquisition, Tracking, and Pointing

Mohammed A. Karim

University of Dayton

Center for Electro-Optics

300 College Park

Dayton, Ohio 45469-0227

513/229-2241 • 513/229-3433

Manuscripts due April 1, 1993.

December 1993

Magnetospheric Imagery and

Atmospheric Remote Sensing

Supriya Chakrabarti

Boston University

Center for Space Physics

725 Commonwealth Avenue

Boston, MA 02215

E-mail: supc@bu-ast.bu.edu

617/353-5990 • 617/353-6463 FAX

Manuscripts due May 1, 1993.

\section{January 1994}

Infrared Technology

Marija S. Scholl

Jet Propulsion Laboratory

California Institute of Technology

4800 Oak Grove Drive

Pasadena, CA 91009-8099

818/354-2313 • 818/393-6105 FAX

Manuscripts due May 1, 1993.

February 1994

Optical Interconnects and Packaging

Sing Lee

University of California/San Diego

E\&CE Department

La Jolla, CA 92093-0407

619/534-2413 • 619/534-1225 FAX

Manuscripts due July 1, 1993.

March 1994

High Heat Flux Optical Engineering

Ali M. Khounsary

Argonne National Laboratory

Advanced Photon Source, APS 362

Argonne, IL 60439

708/252-3384 - 708/252-3222 FAX

Manuscripts due Aug. 1, 1993.

April 1994

Information Processing

Joseph L. Homer

Rome Laboratory

EROP

Hanscom AFB, MA 01731-5000

$617 / 377-3841 \cdot 617 / 377-5041$ FAX

Bahram Javidi

University of Connecticut

School of Engineering

Department of Electrical and Systems Engineering

Room 312, U-157

260 Glenbrook Road

Storrs, CT 06269-3157

203/486-4816 • 203/486-3789 FAX

Manuscripts due Sep. 1, 1993
May 1994

Semiconductor Infrared Detectors

Antoni Rogalski

Military Academy of Technology

Institute of Technical Physics

ul. Apeninska $2 \mathrm{~m} 6$

PL-01-493 Warszawa, Poland

$4822369109 \cdot 4822362254$ FAX

Manuscripts due Oct. 1, 1993.

June 1994

Optical Science \& Engineering in India

Rajpal S. Sirohi

Indian Institute of Technology

Applied Optics Laboratory

Physics Department

Madras-600 036, India

044-2351365 ext. $221 \cdot 044-2350509$ FAX

Manuscripts due Nov. 1, 1993.

July 1994

Adaptive Wavelet Transforms

Harold H. Szu

U.S. Navy

Naval Surface Warfare Center

Code R44

10901 New Hampshire Avenue

Silver Springs, MD 20903-5000

301/394-3097 • 301/394-3923 FAX

Manuscripts due Dec. 1, 1993.

August 1994

Digital Image Recovery and Synthesis

Paul S. Idell

Air Force Phillips Lab.

PL/GPOA

390 B Great Road, \#18

Acton, MA 01720

612/377-3663 • 617/377-3661 FAX

Manuscripts due Dec. 1, 1993.

September 1994

Optics in South Africa

Hannes Markusse

ELOPTRO

Institute of Atomic Physics

P.O. Box 869

Kempton Park 1620, South Africa

Maurice W. McDowell

CSIR/Production Technology Div.

Productiontek

P.O. Box 395

Pretoria 0001, South Africa

$27128413418 \cdot 27128412131$ FAX

Manuscripts due Jan. 1, 1994

November 1994

Micro-Optics

Chandrasekhar Roychoudhuri

University of Connecticut at Storrs

Photonics Research Center

MS-157, Room 312

260 Glenbrook Road

Storrs, CT 06269-3157

203/486-4816 - 203/486-3789 FAX

Manuscripts due April 1, 1994. 